\title{
Effect of increase in Antioxidants Supplementation on Kidney Tissue of Mice Subjected to Fractionated Dose of $\gamma$-Irradiation.
}

\author{
*Hanafi N., **Abel Baset El Aaser, ${ }^{* * * * M o h a m e d ~ S . ~ G a b r y, ~}{ }^{* * *}$ Amany A \\ Tohamy and ***Manal El Saied Ebrahim \\ *Radiation Biology Department, National Centre for Radiation Research and Technology, \\ Atomic Energy Authority, Cairo, Egypt**Cancer Biology Depart. Cancer Institute, Cairo \\ University $* * *$ Zoology Dept. Faculty of Science, Helwan University.
}

\begin{abstract}
Introduction: Although there is little doubt that antioxidants are a necessary component for good health, no one knows if supplements should be taken and, if so, how much.

Materials and methods: To investigate the increase in antioxidants supplementation, a group of mice feeding on a diet containing $20 \%$ soybean was used as control group. The control group treated with 5\% Nigella sativa and Antox drug (3 mg/mouse/day) either alone or in combination for 15 days. Another control group was treated with the same antioxidants and subjected to 1 Gy of $\gamma$ - irradiation $x 5$ times day after day. In kidney tissue the levels of malonaldehyde (MDA) and reduced glutathione (GSH) were estimated. Also, the histopathological changes were recorded.

Results: 5\% Nigella sativa revealed a little decrease in MDA level and a highly significant decrease in GSH in kidney tissue while the exposure to fractionated dose of $\gamma$ irradiation predicted a highly significant decrease in MDA and a highly significant increase in GSH level. Gastric intubations of Antox drug showed a highly significant increase in MDA and GSH levels in kidney tissue either alone or in addition to fractionated radiation exposure. Combined treatments of Nigella sativa and Antox drug showed a highly significant decrease in MDA level and a highly significant increase in GSH level in kidney tissue in comparison to the control group. Histopathological changes showed that treatment with 5\% Nigella sativa and Antox drug either alone or in combination revealed toxic effect on kidney tissue. However, their treatments prior to radiation exposure showed ameliorating effect.

Conclusion: Our findings indicated that we must use the antioxidants under control or under oxidative stress.
\end{abstract}

\section{Introduction}

Antioxidant in a classification of several organic substances, including vitamins $\mathrm{C}, \mathrm{E}$ and vitamin $\mathrm{A}$ (which is converted from beta-carotene), selenium (a mineral), and a group known as the carotenoids. At the molecular and cellular levels, antioxidants serve to deactivate certain particles called free radicals. Antioxidants are widely used as ingredients in dietary supplements that are used for health purposes such as preventing cancer and heart disease. However, many studies have suggested benefits for antioxidant supplements in laboratory experiments, excess supplementation may be harmful. It is logic to assume that one dimensional approach to dietary supplementation with one specific antioxidant is not a panacea, since a broad diet rich in phytonutrients will yield thousands of different polyphenol antioxidants available for metabolism. Also, clinicians should advise their patients against the use of antioxidant dietary supplements during chemotherapy or 
radiotherapy (Gabriella and D'Andrea, 2005). So, the aim of this study is to investigate the effect of supplemented large quantities of antioxidants on the histopathological structure and antioxidant status of the kidney tissue in mice exposed to fractionated dose of $\gamma$ - irradiation.

\section{Material and methods}

40 males of Swiss albino mice each weighing 18-20 g were obtained from the laboratory of experimental of serum and vaccine authority. Irradiation was performed by gamma cell 40 (cesium 137) at the National Centre for Radiation Research and Technology (NCR.RT). Exposure to $\gamma$ irradiation was performed by $1 \mathrm{~Gy}$ of $\gamma$ irradiation exposure / day x 5 .

The animals were fed on a diet containing $20 \%$ soy bean (control diet).Others fed on a diet containing 20\% soy bean and 5\% Nigella sativa. They also maintained on nutritional form of drug (Antox) purchased from Arab Company for Pharmaceuticals and Medicinal Plants (MEPACO) contains the three main antioxidant vitamins $(\mathrm{A}, \mathrm{C}$, and $\mathrm{E})$ together with selenium. All the animals were maintained under the experimental conditions for 15 days.

\section{Groups of animals under investigation:} The experimental animals were divided as following:

G I: A group of 5 animals fed on a diet containing $20 \%$ soy bean (control diet) for 15 days.

G II: A group of 5 animals fed on the control diet and 5\% Nigella sativa for 15 days.

GIII: A group of 5 animals fed on the control diet and orally supplemented with $3 \mathrm{mg} / \mathrm{mouse} /$ day of Antox drug for 5 times, every other day

GIV: A group of 5 animals fed on the control diet and 5\% Nigella sativa and orally supplemented with $3 \mathrm{mg} / \mathrm{mouse} /$ day, of Antox drug for 5 times every other day.

$\mathrm{GV}$ : A group of 5 animals fed on the control diet and exposed to 1 Gy of $\gamma$ - irradiation $\times 5$ times every other day.

GVI: A group of 5 animals fed on the control diet and 5\% Nigella sativa and exposed to $1 \mathrm{~Gy}$ of $\gamma$ irradiation exposure / day x 5 .

GVII: A group of 5 animals fed on the control diet and orally supplemented with $3 \mathrm{mg} / \mathrm{mouse} /$ day of Antox drug $x 5$ times every other day and exposed to $1 \mathrm{~Gy}$ of $\gamma$ - irradiation after two hours of Antox administration.

GVIII: A group of 5 animals fed on the control diet and 5\% Nigella sativa and orally supplemented with $3 \mathrm{mg} /$ mouse/day of Antox drug for 5 times every other day and exposed to 1 Gy of $\gamma$-irradiation after two hours of Antox administration.

After the end of the experiment samples of nearly $1 \mathrm{~cm}^{3}$ of kidney tissue were removed from the dissected animals. Part of tissue was stored in refrigerator for biochemical analysis and the other part was fixed in $10 \%$ neutral formalin for histopathological studies.

For histopathological studies, tissue sections stained with Hx. and E. stain. For biochemical estimations, malonaldehyde (MDA) estimated according to the Method of Ohkhawa et al. (1979) and Glutathione content (GSH) was determined by the method reported by Beutler et al. (1963).

\section{Results}

\section{Biochemical Estimation.}

Fig. (1) represents the effect of antioxidants on MDA levels in kidney tissue of mouse exposed to fractionated dose of $\gamma$-irradiation.

Feeding of the experimental mice on the control diet and 5\% Nigella sativa revealed some decrease in MDA levels in kidney tissue in compared to the control group. The animals treated with (3 $\mathrm{mg} / \mathrm{mouse} /$ day) of Antox drug showed a very highly significant increase in MDA level $(\mathrm{P}<0.01)$ in comparison to control group. Treatment of experimental animals 
with the control diet and 5\% Nigella sativa and the gastric intubations of them with Anotox drug indicated a highly significant decrease in MDA levels in kidney tissue in comparison to control group.

The level of MDA was decreased significantly due to the effect of exposure of animals to fractionated dose of $\gamma$-irradiation. While the level of MDA in animals fed on the control diet and 5\% Nigella sativa and exposed to fractionate dose of $\gamma$ irradiation displayed highly significantly decrease in comparison to control group and the irradiated one. On the other hand on treatment with Antox drug and exposure to fractionated dose of $\gamma$-irradiation, a highly significant increase in MDA level was observed in compared to the control and irradiated groups. Whenever feeding on the control diet and 5\% Nigella sativa, gastric intubations of $3 \mathrm{mg} / \mathrm{mouse} /$ day of Antox drug and the exposure to $\gamma$-irradiation recorded a very highly significant decrease in MDA level in kidney tissue in comparison to the control and irradiated groups.

From fig (2) we can evaluate the effect of antioxidants on GSH levels in kidney tissue of mouse exposed to fractionated dose of $\gamma$-irradiaion.

Feeding of experimental mice on the control diet and 5\% Nigella sativa revealed a highly significant decrease in GSH level in kidney tissues. While when the experimental animals were treated with (3 $\mathrm{mg}$ /mouse/day) of Antox drug in addition to the control diet a very highly significant increase in GSH level was represented $(\mathrm{P}<$ 0.001 ) in comparison to control group. Feeding on the control diet and 5\% Nigella sativa and gastric intubations of Antox drug recorded a very highly significant increase $(\mathrm{P}<0.001)$ in GSH level of kidney tissue when compared to control group. The exposure of experimental animals to fractionated dose of $\gamma$-irradiation showed a very highly significant increase in GSH level in kidney tissue. When the animals were fed on the control diet , 5\% Nigella sativa and exposed to fractionated dose of $\gamma$-irradiation a very highly significant increase in GSH level was observed in kidney tissue. Feeding on the control diet, 5\% Nigella sativa, oral administration of Antox drug and the exposure to fractionated dose of $\gamma$ irradiation a very highly signifi-cant increase in GSH level in kidney tissue occurred in comparison to control group.

\section{Histopathological observations.}

Fig (3) represents the histopathlogical appearance of mouse kidney tissue as affected by excess antioxidants. Fig.(3-1) reveals normal histological appearance of the kidney tissue section of mouse fed on the control diet (containing 20\% soybean) . The kidney tissue shows normal appearance composed of renal Malpighian corpuscles $(\downarrow \downarrow)$, proximal convoluted tubules (blocked arrow) and distal convoluted tubules (arrow head). Fig.(3-2) illustrates the histopathological changes in kidney tissue when the experimental animals fed on the control diet and 5\% Nigella sativa. The convoluted tubules showed protrusions in the inner surface. Infiltration of some red blood cells in addition to the presence of shrunken glomerulus $(\downarrow \downarrow)$ were also recorded. Feeding of the experimental animals on the control diet and treating of them with $3 \mathrm{mg} /$ mouse/day of Antox drug for 5 times every other day, more thicken on the inner surface of the convoluted tubules (congestion) ( $\uparrow$ ) in addition to the presences of shrunken glomerulus and the absence of juxtaglomerular apparatus (curved arrow) were observed (fig.3-3). Treatment of the control group with 5\% Nigella sativa and the gastric intubations with Anotox drug showed highly structural changes in kidney tissue. A marked damage in convoluted tubules $(\downarrow)$ was noticed and the presence of internal glumerular capsule bleeding (blocked arrow) (fig.3-4) was also predicted.

No histopathological observations were noticed when control group was subjected to fractionated dose of $\gamma$-irradiation exposure except thin fibroid area lining the inner surface of the Bowman's capsule $(\downarrow \downarrow)$ (fig.4-5). When the control group was treated with 5\% Nigella sativa and exposed to fractionated dose of $\gamma$-irradiation, infiltration of some reed blood cells in addition to the presence of shrunken glomeruli ( $\downarrow$ ) were observed (fig.4-6). 


\section{Hanafi N et al}

However, the gastric intubations of the experimental animals with Antox drug and exposure of them to fractionated dose of $\gamma$ irradiation, the glumerulus showed lobulated shape ( $\leftarrow$ ) (fig.4-7). Return to normal appearance of the kidney tissue structure was observed when the experimental animals fed on a diet containing 20\% soybean and 5\% Nigella sativa, gastric intubated with Anotox drug Antox drug and exposed to fractionated dose of $\gamma$-irradiation ( fig.4-8).

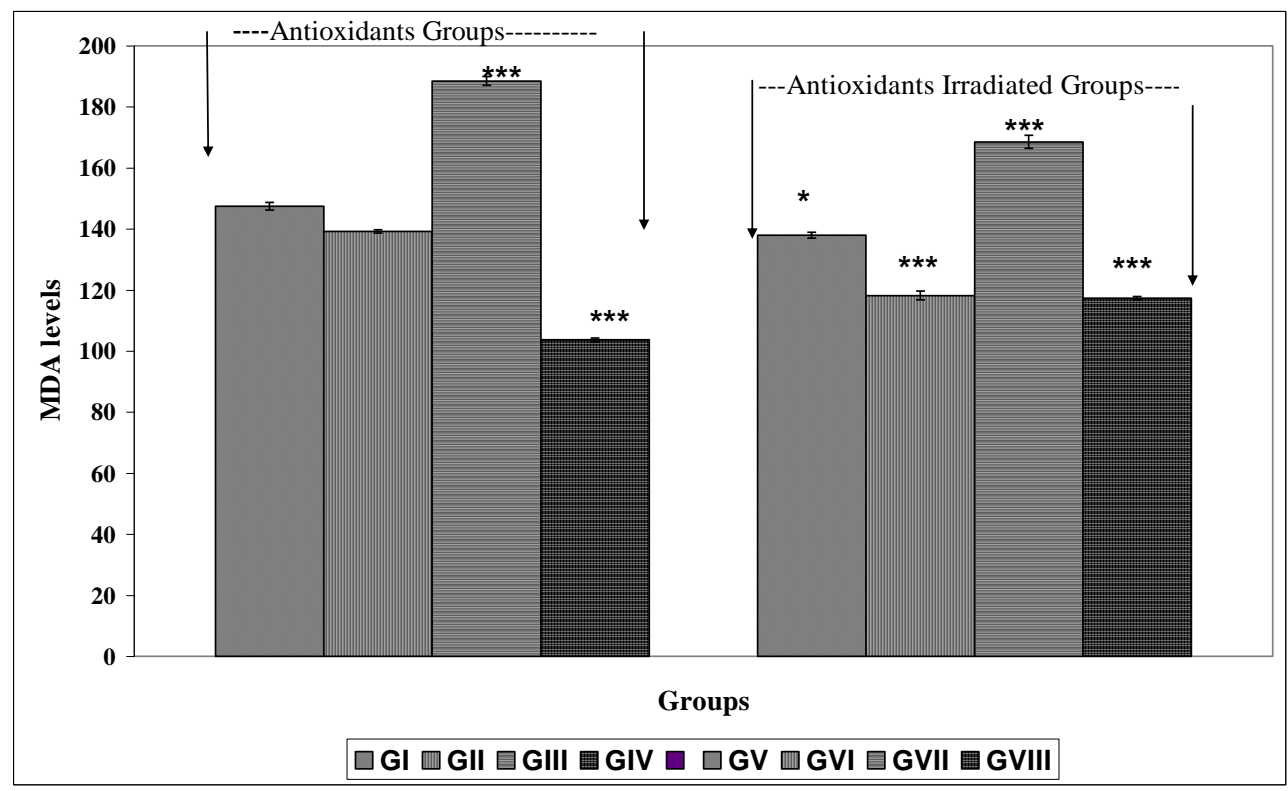

Fig (1): Effect of antioxidants on MDA levels.

Values are the mean of 5 observations.

$* \mathbf{p}<0.05 * * * \mathbf{p}<0.001$ in comparison to control group.

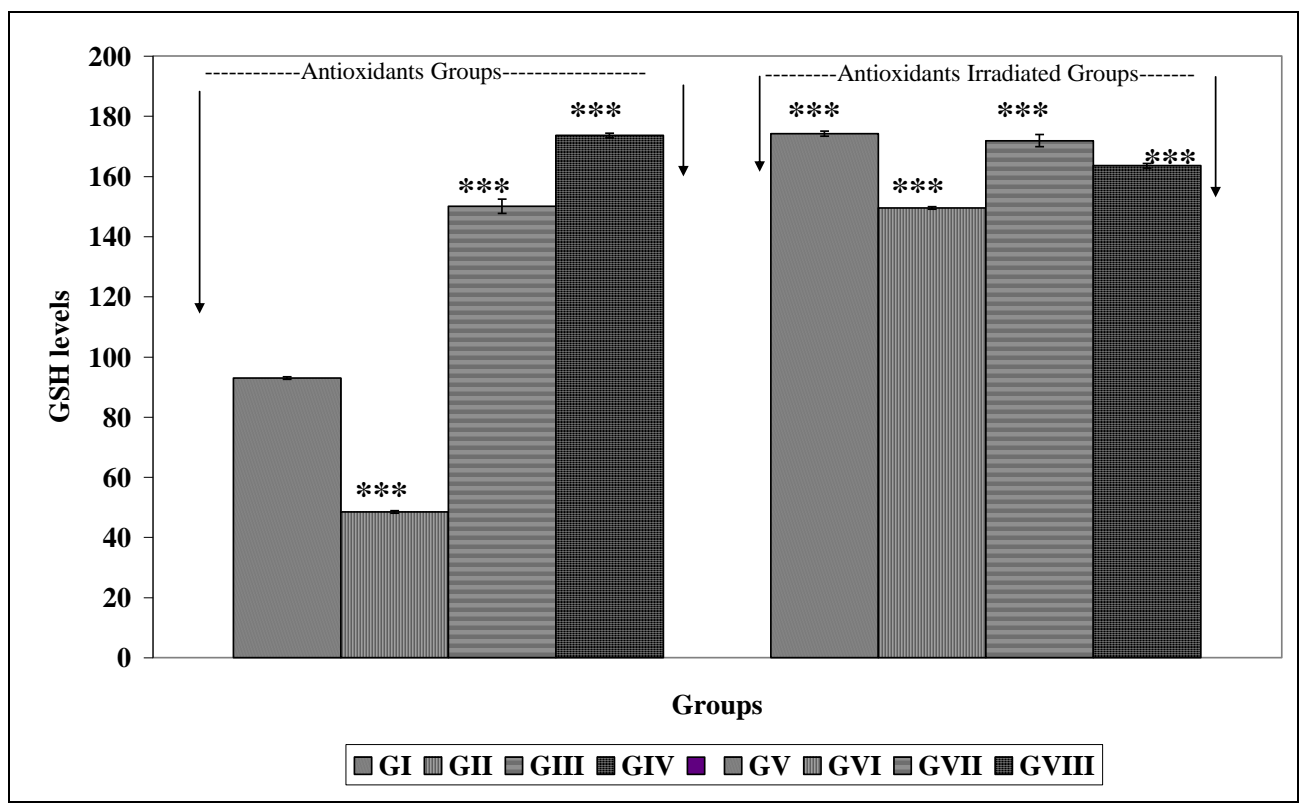

Fig (2): Effect of antioxidants on GSH levels.

Legends as in fig. (1) 


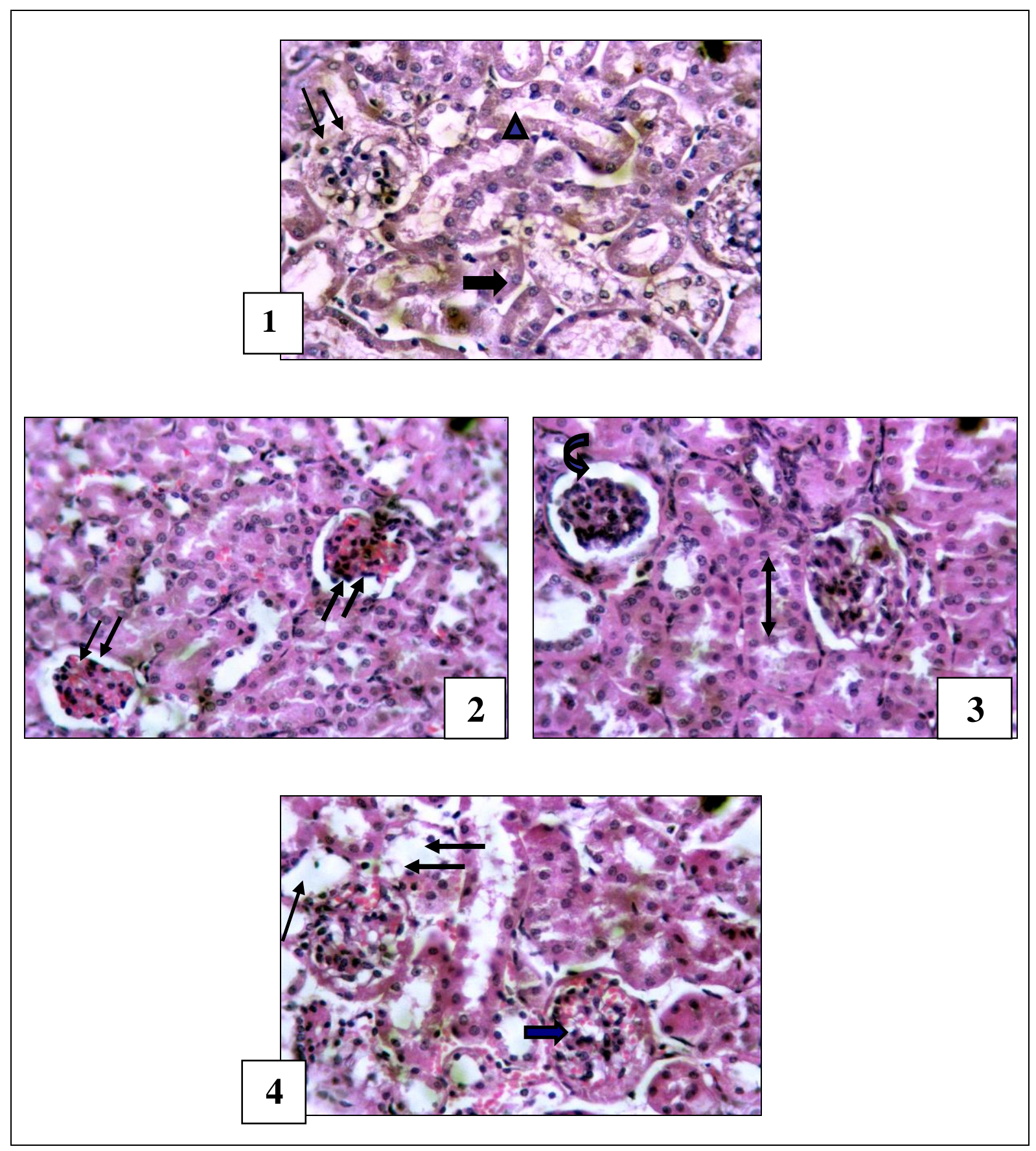

Fig(3): Section in kidney of mice as affected by different antioxidants.All of them stained with Hx.\&E.and magnification at $\mathbf{x 4 0 0 . ( 1 ) ~ S e c t i o n ~ i n ~ k i d n e y ~ o f ~ m o u s e ~ f e e d i n g ~ o n ~}$ control diet.(2) Section in kidney of mouse treated with Nigela sativa.(3) Section in kidney of mouse treated with Antox drug.(4) Section in kidney of mouse treated with Nigela sativa and Antox drug. 


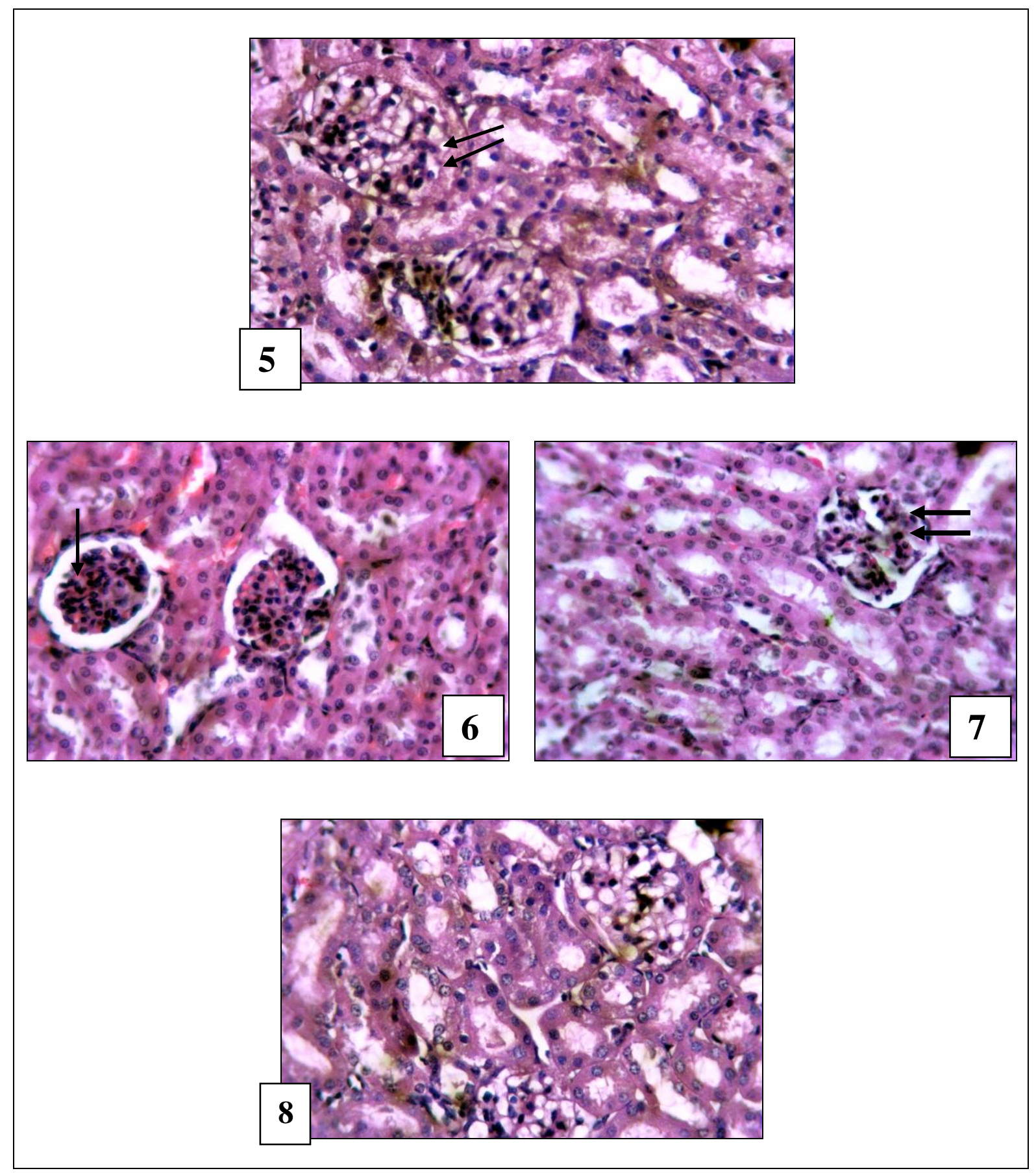

Fig(4): Section in kidney of mice as affected by different antioxidants and exposed to fractionated dose of $\gamma$-irradiation .All of them stained with Hx.\&E.and magnification at $x 400$.(5) Section in kidney of mouse exposed to fractionated of $\gamma$ irradiation .(6) Section in kidney of mouse treated with Nigela sativa exposed to fractionated of $\gamma$-irradiation.(7) Section in kidney of mouse treated with Antox drug and exposed to fractionated of $\gamma$-irradiation.(8) Section in kidney of mouse treated with Nigela sativa, Antox drug and exposed to fractionated of $\gamma$ irradiation. 


\section{Discussion}

SportsMedWeb (1995) published that there is little doubt that antioxidants are a necessary component for good health. No one knows if supplements should be taken and, if so, how much. Antioxidants supplements were once thought to be harmless but increasingly we are becoming aware of their interactions and potential toxicity. It is interesting to note that, in the normal concentrations, vitamin $\mathrm{C}$ and betacarotene are antioxidants; but at higher concentrations they are pro-oxidants and, thus, harmful. Also, very little is known about the long-term consequences of megadoses of antioxidants. The body's finely tuned mechanisms are carefully balanced to withstand a variety of insults. Taking chemicals without a complete understanding of all of their effects may disrupt this balance.

In the current study experimental animals fed on the control diet (containing $20 \%$ soybean) and 5\% Nigella sativa, no pronounced decrease in MDA level in kidney tissue in addition to a highly significant decrease in GSH level was observed.

Mahmoud et al. (2001) reported that thymoquinone the main constituent of Nigella sativa didn't influence reduced glutathione content in kidney tissue.

Treatment of the experimental animals with Antox drug predict a highly significant increase in MDA and GSH levels in kidney tissue which illustrate the disturbance in the antioxidant status in the kidney tissue.

Under normal condition, excess of antioxidants in blood produces toxic radicals which can be uptakes by various tissue recycling mechanisms, and oxidizedlow density lipoprotein (LDL) particles that may form are rapidly cleared by the liver (Steinberget et al., 1989). In contrast, the same antioxidants-derived radicals accumulate within the vessel wall and may not be as readily cleared, resulting in a prooxidative effect.

Heart Protection Study Collaborative Group (2002) and Yusuf (2002) reported that the increase and long term in antioxidants supplementation therapy significantly increases the blood levels of LDLcholesterol and triglycerides, which have the potential to increase cardiovascular disease risk.

Previous finding, were also illustrated by the histopathological observations where the shrunken glumeruli and congestion in convoluted tubules were observed.

Exposure to fractionated dose of $\gamma$ irradiation predicts significant decrease in MDA level and significant increase in GSH level but combined by non pathological changes in kidney tissue because low doses of ionizing radiation exposure stimulate various biological functions like antioxidative capacity (Kojima et al.,1998), DNA repair capability (Le et al., 1998), apoptosis (Cregan et al., 1999 ; Sakai.2000) and immune functions (Nogami et al.,1993 and Nogami et al., 1994) which would be initiated with DNA damage induced directly by radiation or through reactive oxygen species production(Sakai et al.,2004).

Significant decrease in kidney tissue MDA level accompanied by highly significant increase GSH level was observed when the experimental animals treated by 5\% Nigella sativa prior to the exposure through its immunomodulator and antioxidant activities (Turkdogan et al., 2001). However the histopathological observations revealed some glomerulus changes.

Supplementation of Antox drug prior to $\gamma$-irradiation exposure leads to highly significant increases in MDA and GSH levels. Cao and Cutler (2006) reported that high concentrations of antioxidants which increase the GSH level may not improve defense against oxidative stress which increase the MDA level.

In the current study highly histopathological changes were recorded in kidney tissue after treatment of the experimental animals with Nigella sativa and gastric intubations of Antox drug which illustrate the toxic effect of increasing antioxidants supplementation under normal 
conditions. However the biochemical observations showed ameliorations by the addition of 5\% Nigella sativa and /or fractionated dose of $\gamma$-irradiation exposure to Antox drug by the immunomodulator and antioxidant activities of Nigella sativa (Turkdogan et al., 2001). For that individuals should avoid use of antioxidants unless they are under the close supervision of a physician.

\section{References}

1. Beutler E, Duron O. and Kelly B. (1963): Improvemed method for determination of blood glutathione. M. J. Lab. Clin. Med., 61 (5): 882

2. Cao G. and Cutler R G. (2006): High concentrations of antioxidants may not improve defense against oxidative stress Molecular Physiology and Genetics Section, Laboratory of Cellular and Molecular Biology, Gerontology Research Center, National Institute on Aging, National Institutes of Health, 4940 Eastern Avenue, Baltimore, MD 21224, USA.

3. Cregan SP., Brown DL. and Mitchel RE. (1999): Apoptosis and the Adaptive Response in Human Lymphocytes. Int. J. Radiat. Biol.75:1087-.1094

4. Daniele V., Elena D. and Martin RP. et al., (2006): Chronic Antioxidant Supplementation Impairs Coronary Endothelial Function and Myocardial Perfusion in Normal Pigs Hypertension.,47:475-481.

5. Gabriella M. and D'Andrea M D. (2005): Use of antioxidants during chemotherapy and radiotherapy should be avoided. Cancer J. Clin., 55:319-321.

6. Heart Protection Study Collaborative Group (2002): MRC/BHF Heart Protection Study of antioxidant vitamin supplementation in 20,536 high-risk individuals: a randomized placebo-controlled trial. Lancet, 360: 23-33.

7. Kojima S., Matsuki O.and Nomura T. et al., (1998): induction of mrnas for glutathione synthesis-related proteins in mouse liver by low doses of gamma-rays. Biochim. Biophys. Acta 1384:312-318.

8. Le X C., Xing JZ., Lee J., Leadon SA. and Weinfeld M. (1998): inducible repair of thymine glycol detected by an ultrasensitive assay for DNA damage. Science, 280:1066-1069

9. Mahmoud A., Mahmoud N., Nagi, Aiman S. and Abdullah M. (2001): Effects of thymoquinone on antioxidant enzyme activities, lipid peroxidation and DT-diaphorase in different tissues of mice: a possible mechanism of action. Cell Biochemistry and Function, 20 (2): 143 151.

10. Nogami M., Huang JT., Nakamura LT. and Makinodan T (1994): $T$ cells are the cellular target of the proliferation-augmenting effect of chronic low-dose ionizing radiation in mice. Radiat. Res., 139:48-52.

11. Nogami M., Huang JT., James SJ., et al.,(1993): Mice chronically exposed to low dose ionizing radiation possess splenocytes with elevated levels of hsp70 mrna, hsc70 and hsp72 and with an increased capacity to proliferate. Int. J. Radiat. Biol., 63:775-783.

12. Ohkhawa H., Ohishi N. and Yogi K.(1997): Assay for lipid peroxidation in animals tissue by thiobarbituric acid reaction. Anal Biochem., 95:351-358.

13. Sakai K. (2000): Effects of low-dose pre irradiation on radiation-induced cell death in cultured mammaliancells. biological effects of low dose radiation., Excerpta Medica International Congress Series 1211: 53-58.

14. Sakai K., Takaharu N. and Yasuhiro I. (2004): Enhancement of bio-protective functions by low dose/dose-rate radiation. Low Dose Radiation Research Center, Central Research Institute of Electric Power Industry2-11-1 Iwado-kita, Komae, Tokyo, Japan, 8201-8511.

15. Sports Med Web (1995): Prospects for the use of antioxidant therapies. (Review). Drugs, 49(3):345-361.

16. Steinberg D., Parthasarathy S., Carew TE., et al., (1989): Beyond cholesterol. Modifications of low-density lipoprotein that increase its atherogenicity. N. Engl. J. Med., 320:915-924.

17. Turkdogan MK., Agaoglu Z., Yener Z., et al., (2001): The role of antioxidant vitamins ( $\mathrm{C}$ and $\mathrm{E}$ ), selenium and Nigella sativa in the prevention of liver fibrosis and cirrhosis in rabbits: new hopes. Dtsch Tierarztl Wochenschr; 108: 71-73.

18. Yusuf S. (2002): Two decades of preventing vascular disease. Lancet, 360: 2-3. 


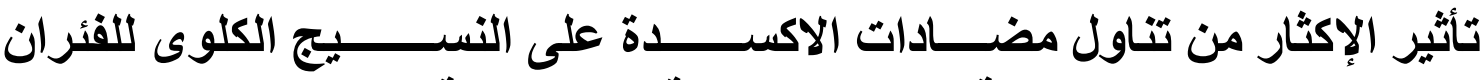

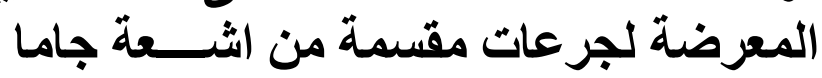

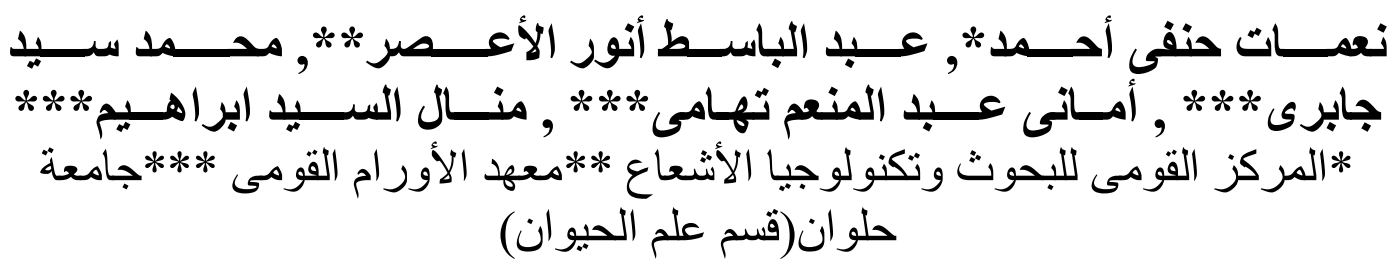

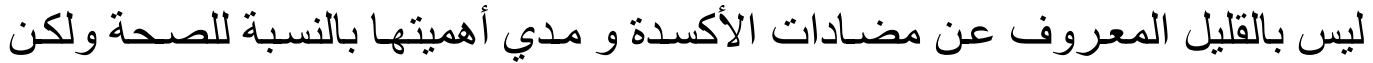

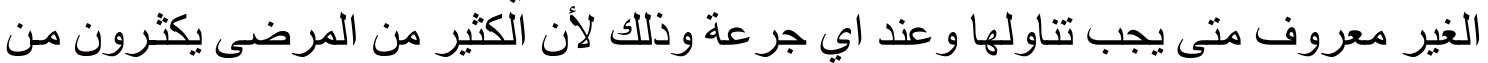

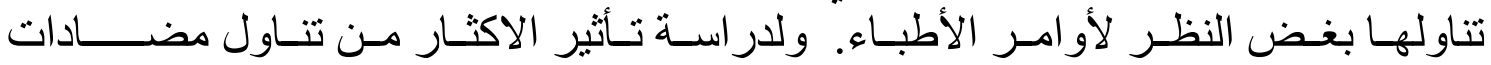

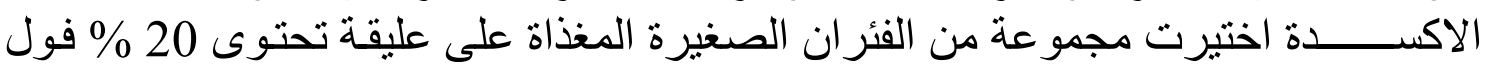

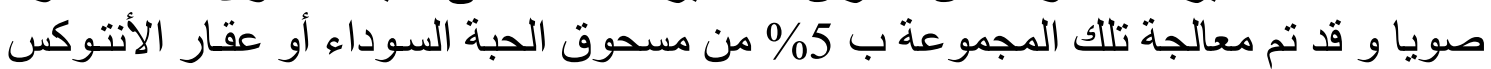

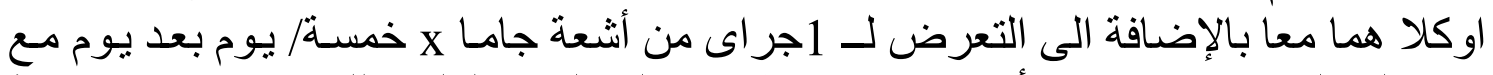

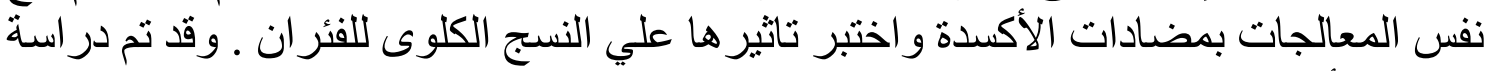

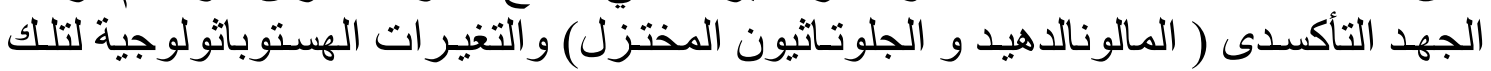

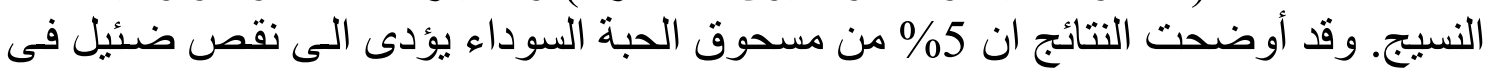

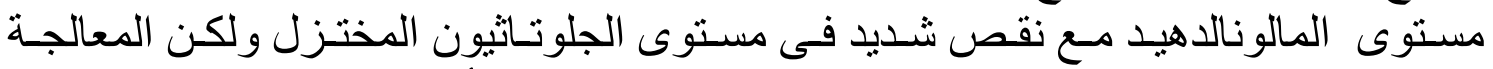

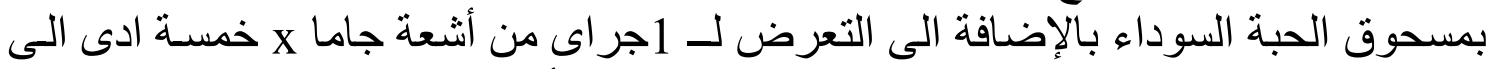

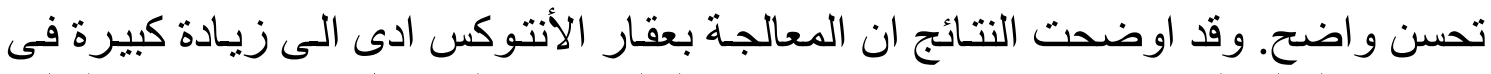

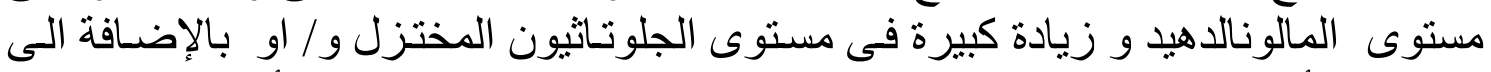

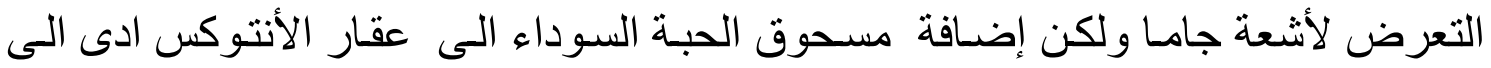

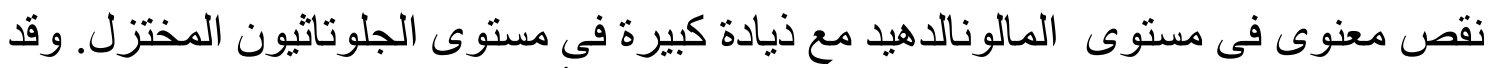

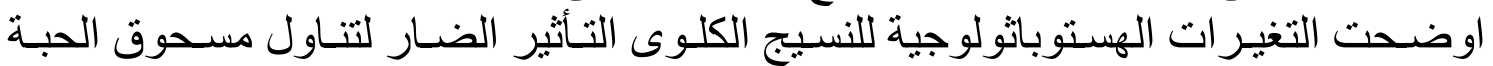

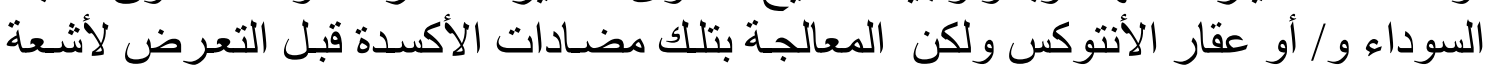

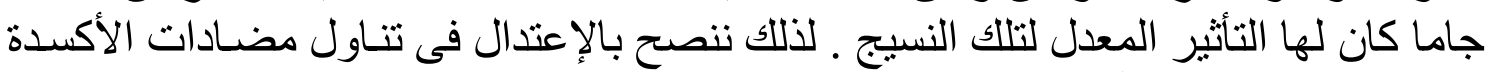

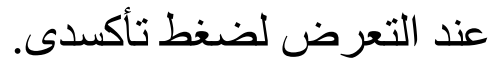

